\title{
Prognostic impact of clinical, haematological, and histopathological variables in 102 canine cutaneous perivascular wall tumours
}

Prognostic factors in canine PWT

Chiti $\mathrm{LE}^{1}$, Ferrari R (corresponding) ${ }^{1}$, Boracchi $\mathrm{P}^{2}$, Morello $\mathrm{E}^{3}$, Marconato $\mathrm{L}^{4}$, Roccabianca $\mathrm{P}^{1}$, Avallone $\mathrm{G}^{4}$, Iussich $\mathrm{S}^{3}$, Giordano $\mathrm{A}^{1}$, Ferraris $\mathrm{EI}^{3}$, Agnoli $\mathrm{C}^{4}$, Dondi $\mathrm{F}^{4}$, Giacobino $\mathrm{D}^{3}$, Godizzi $\mathrm{F}^{1}$, Stefanello $\mathrm{D}^{1}$

${ }^{1}$ Dipartimento di Medicina Veterinaria, Università degli Studi di Milano, Milan, Italy

${ }^{2}$ Dipartimento di Scienze Cliniche e di Comunità, Laboratorio di Statistica Medica, Biometria ed Epidemiologia “G.A. Maccacaro”, Università degli Studi di Milano, Milan, Italy

${ }^{3}$ Dipartimento di Scienze Veterinarie, Università degli Studi di Torino, Turin, Italy

${ }^{4}$ Dipartimento di Scienze Mediche Veterinarie (DIMEVET), Università degli Studi di Bologna, Ozzano dell'Emilia, Italy

Corresponding Author:

Dr. Roberta Ferrari

Dipartimento di Medicina Veterinaria, Università degli Studi di Milano

Via dell’Università 6, 26900 - Lodi (Italy)

Email: roberta.ferrari@unimi.it

Data availability statement: The data that support the findings of this study are available from the corresponding author upon reasonable request.

Conflict of interest: Authors declare the absence of conflict of interest.

This article has been accepted for publication and undergone full peer review but has not been through the copyediting, typesetting, pagination and proofreading process which may lead to differences between this version and the Version of Record. Please cite this article as doi: $10.1111 /$ vco.12673 


\section{Abstract}

Identification of prognostic factors for perivascular wall tumours (PWTs) is desirable to accurately predict prognosis and guide treatment. One-hundred and two dogs with surgically excised PWTs without distant metastasis were retrospectively enrolled in this multi-institutional study, and the impact of pre-treatment leukocyte parameters, clinical and histopathological variables on local recurrence (LR) and overall-survival time (OST) were evaluated.

Increasing values of white blood cell count (WBCC), neutrophil count (NC) and neutrophil-tolymphocyte ratio (NLR) were significantly correlated with the hazard of LR in univariate analysis. WBCC and NC remained prognostic when adjusted for margins, grade, tumour size, location and skin ulceration, but lost their significance when adjusted for mitotic index and necrosis, while NLR remained prognostic only when close margins where categorized as infiltrated.

Castrated males had a higher hazard of LR than intact males in univariate analysis, but significance was lost in multivariate models. Ulcerated PWTs and those located on the distal extremities had a higher hazard of LR both in univariate and multivariate analysis. Histological grade, necrosis, mitotic count, and infiltrated margins were all associated with LR both in univariate and multivariate analysis. Boxer breed, older age, ulceration, grade III, necrosis $>50 \%$ and higher mitotic count were correlated with shorter OST, although breed and age lost their significance in multivariate analysis. Prognostication of surgically excised PWTs should be based on both clinical and histopathological variables. If validated in further studies, leukocyte counts and NLR may aid the clinician in identifying dogs at higher risk of LR before treatment.

\section{Key words}

Dog, soft tissue sarcoma, surgery, oncology, neutrophil-to-lymphocyte ratio 


\section{Introduction}

Perivascular wall tumours (PWTs) are a subgroup of canine soft tissue sarcomas (STSs), characterised by specific histologic and immunophenotypic patterns that tend towards a more benign clinical behaviour compared with other STSs. ${ }^{1-3}$ Pulmonary metastases are rarely reported, and rates of local recurrence (LR) are relatively low, with one paper estimating a probability of being free from LR of $98 \%$ at six months, $92 \%$ at one year, $80 \%$ at two years and $76 \%$ at three years. ${ }^{2}$ However, the definition of prognostic factors for PWTs is desirable to identify the subpopulation of dogs at risk for tumour relapse that may benefit from a higher surgical dose or adjuvant treatment. ${ }^{4}$ Two previous studies have investigated the prognostic impact of clinical and histological variables on 55 and 56 surgically excised PWTs. ${ }^{2,3}$ Tumour size and depth have been correlated with a higher hazard of LR, while histological grading, which is a well-established prognostic factor for STSs does not seem to play a role for PWTs. ${ }^{2,3}$ To date, however, the majority of prognostic studies have been conducted on the heterogeneous group of STSs, and prognostic information on a large population of PWTs is still lacking. ${ }^{4}$

The role of the systemic inflammatory response against cancer is widely accepted in human oncology and is becoming increasingly evident in small animal oncology as well. ${ }^{5}$ Indeed, inflammation orchestrates tumour microenvironment, which is crucial for proliferation, survival and migration of cancer cells. ${ }^{6}$ Thus, there is a growing interest in the identification of inflammatory markers as prognostic factors for several malignancies. Pre-treatment neutrophil-to-lymphocyte ratio (NLR) is a useful prognostic tool for several malignancies in human medicine, including STSs. $^{7}$ Similarly, the veterinary literature has recently investigated the potential diagnostic or prognostic role of several leukocyte counts and ratios, including NLR, for canine lymphoma, osteosarcoma, mast cell tumour (MCT), STS, ${ }^{8-12}$ and feline injection-site sarcoma (FISS), ${ }^{13}$ with promising results.

This multicenter study aims to retrospectively assess the impact on time to LR (TLR) and overall survival time (OST) of pre-treatment NLR and leukocyte counts in dogs undergoing curative-intent 
excision of cutaneous PWTs at first presentation. Given the paucity of prognostic studies focusing on PWTs, the prognostic role of additional clinical and histopathological variables was evaluated on a large cohort of dogs.

\section{Materials and methods}

Medical records from three veterinary teaching hospitals were searched for client-owned dogs diagnosed with cutaneous PWTs between January 2001 and December 2019. Included dogs fulfilled the following criteria: first occurrence and histologically confirmed PWT; absence of distant metastasis (confirmed by pre-operative total-body contrast-enhanced CT or thoracic X-rays and abdominal ultrasound); curative-intent surgical excision; availability of pre-treatment leukocyte counts (within 45 days before surgery). We excluded dogs that had undergone neoadjuvant chemotherapy or radiotherapy, those receiving corticosteroids or antibiotics within two months before surgery or presenting with Cushing's syndrome.

Recorded data included: signalment, tumour characteristics (location, clinical size at longest diameter, ulceration), presence of concomitant diseases, status of regional lymph nodes (if assessed), type of surgery, histopathological findings and adjuvant treatment (if any).

Complete blood cell counts (CBC) with leukocyte differential were performed on blood samples collected in EDTA, within 24 hours from sampling. Haematological analyses were performed with the same laser-based analyser at the laboratories of the three institutions participating in the study (ADVIA®120 Hematology System, Siemens Diagnostics), and differentials were confirmed microscopically on May-Grünwald-Giemsa stained blood smears. Alterations of the normal values of white blood cell count (WBCC), neutrophil count (NC) and lymphocyte count (LC) were defined based on the reference intervals of the laboratories. NLR was determined by the ratio of NC to LC. Surgical excision was classified as marginal if the surgical margin was adjacent to the pseudocapsule, wide if the tumour was excised with $2-3 \mathrm{~cm}$ of macroscopically healthy tissue laterally and one deep fascial plane, and radical if the entire anatomical compartment was resected. The surgical 
approach was decided by the attending surgeon based on tumour location and size, in order to obtain the widest excisional margin without impairing the function of the involved anatomical compartment, if possible. ${ }^{2,14,15}$

The histopathological diagnosis was based on the presence of spindle to polygonal or stellated cells and vascular growth patterns consistent with PWT (perivascular whorls, staghorn vessels, placentoid and medial/intimal bundles). ${ }^{1,2,4}$ Immunohistochemistry was added if needed to obtain definitive diagnosis. ${ }^{1}$ Histopathological variables including grade, ${ }^{16}$ mitotic count in $10 \mathrm{HPF}$ (area of view $2,37 \mathrm{~mm}^{2}$ ), percentage of necrosis (absent, $<50 \%,>50 \%$ ), pattern of tumour growth (expansile or infiltrative), and completeness of surgical margins were recorded.

Surgical margins were evaluated by radial sectioning ${ }^{17}$ and were categorised into tumour-free (histologic tumour free margin $[\mathrm{HTFM}]>3 \mathrm{~mm}$ ), close (HTFM 1-3mm) and infiltrated (neoplastic cells extending to the margin).

Follow-up evaluation was done by serial clinical examinations (approximately every three months) for the first two years after surgery, and by telephone calls to the owner or referring veterinarian thereafter. Time to local recurrence was defined as the interval between the date of surgery and the cytological/histological diagnosis of PWT growing within $2 \mathrm{~cm}$ from the scar of the previous excision. Overall survival time was calculated from the date of surgery to the date of euthanasia or death, further classified as tumour related or unrelated. Dogs lost to follow-up were censored at the date of the last follow-up.

\section{Statistical analysis}

Quantile regression modelling on the median was used to evaluate the association between WBCC, NC, LC, NLR (dependent variables) and each one of the clinical and pathological variables (independent variables). The standard least square regression model was not applied since the distributions of the haematological variables were not Gaussian. ${ }^{18}$ The null hypothesis of no 
association (regression coefficients equal to zero) was tested by $\mathrm{T}$ statistics for each regression coefficient and by F statistics for the overall association.

Median follow-up was calculated with the reverse Kaplan-Meier method. ${ }^{19}$ Overall survival probability was estimated by the Kaplan-Meier method, and univariate analysis was performed by Cox regression model on the hazard of death. A method for competing risks was applied for TLR analysis since death could prevent the observation of LR. Crude cumulative incidence was reported, and univariate analysis on (sub-distribution) hazard of LR was performed by Fine and Gray regression model. ${ }^{20}$ Wald statistics tested the null hypothesis of hazard ratio equal to one for each regression coefficient and overall variable effect.

Given the low number of events, it was not possible to include all the examined variables in multivariate analysis and a model selection procedure could not be applied. ${ }^{21}$ Only a preliminary multivariate analysis was possible for LR, with a maximum number of 4 variables. ${ }^{22}$ Different models were used to evaluate the prognostic role of haematological variables adjusted for clinical and pathological variables. Models were performed, including each one of the haematological variables jointly with the other three variables, selected according to clinical relevance. The same modelling strategy was applied for OST.

Statistical analyses were performed with a software package - quantreg, survival and cmprsk (Rsoftware; www.r-project.org). The significance level was set at $5 \%$.

\section{Results}

A total of 102 dogs fulfilled the inclusion criteria. In 6 cases immunohistochemistry was added to achieve the definitive diagnosis of PWT. The main clinical and histopathological characteristics of the study population are reported in Tables S1A and S1B.

Peripheral blood analyses were performed within a median of 14 days before surgery (range $0-38$ days). White blood cell counts abnormalities were detected in 26 dogs (25\%) and included leukocytosis $(n=12)$, leukopenia $(n=9)$, neutrophilia $(n=7)$, neutropenia $(n=1)$, and lymphopenia 
$(\mathrm{n}=7)$. Median NLR was 3.4 (range 0.3 - 3.8) (Table S1B). Forty-nine dogs (48\%) presented with concomitant diseases (Table S1A).

Regional lymph nodes were clinically normal in all dogs and were thus not sampled. Surgical excision of the PWT was marginal in $59(58 \%)$ dogs, wide in $35(34 \%)$ and radical in $8(8 \%)$ dogs. Adjuvant treatments were administered to 21 (21\%) dogs and included metronomic chemotherapy $(n=11)$, radiation therapy $(n=6)$, radiation therapy and metronomic chemotherapy $(n=2)$, electrochemotherapy $(n=1)$, and doxorubicin followed by metronomic chemotherapy $(n=1)$.

The median follow-up was 705 days (range, 14 to 1996 days). Overall, twenty-nine dogs (29\%) experienced tumour relapse or progression: 19 had LR, 6 had both LR and distant metastases, and 4 had distant metastases alone. The first LR was observed at 25 days, and the last at 1496 days. Distant metastases were observed between 60 and 1060 days postoperatively and were located to lung $(n=7)$, lymph nodes $(n=2)$, skin $(n=1)$, rib $(n=1)$, and peritoneum $(n=1)$. In a dog with pulmonary metastasis a cerebral involvement was also suspected due to suddenly onset of seizure. Considering the competing risk of death, $10 \%$ dogs experienced LR within six months after surgery (95\% C.I. $4 \%-17 \%), 18 \%$ within 1 year (95\% C.I: $10 \%-26 \%)$ and $27 \%$ within 2 years (95\% C.I: $17 \%-38 \%$ ) (Figure 1).

Fifty-six dogs were alive at the end of the study, four were lost to follow-up at 15, 30, 210 and 595 days respectively and 42 died. Cause of death was tumour-related in 13 dogs (6 had LR, 2 experienced distant relapse and 5 had concomitant local and distant relapse). Median OST was 1125 days, with a survival of $82 \%$ at one year, of $66 \%$ at two years and of $51 \%$ at three years (Figure 2).

\section{Association between variables}

Results of the association between leukocyte counts, NLR and clinical/pathological variables are detailed in Table S2.

Breed and sex were associated with WBCC $(\mathrm{p}<0.01 ; \mathrm{p}=0.02)$ and $\mathrm{NC}(\mathrm{p}<0.01 ; \mathrm{p}=0.01)$. 
Tumour grade and necrosis were associated with $\mathrm{NC}(\mathrm{p}=0.01 ; \mathrm{p}<0.01)$ : median $\mathrm{NC}$ values were higher in grade III than in grade I PWT $(\mathrm{p}=0.03)$ and in tumours with $>50 \%$ necrosis than in those with $<50 \%$ necrosis $(\mathrm{p}<0.001)$. Median LC values were associated with tumour location $(\mathrm{p}<0.01)$ and were higher in intact males than intact females $(\mathrm{p}=0.03)$. NLR was not associated with any of the examined variables.

All leukocyte counts and ratios were associated with one another, except for LC and NC.

\section{Prognostic impact of variables on TLR}

In univariate analysis (Table 1), WBCC $(\mathrm{p}<0.001)$, NC $(\mathrm{p}<0.001)$ and NLR $(\mathrm{p}=0.044)$ had a significant impact on LR, while LC did not $(\mathrm{p}=0.279)$. The hazard of experiencing $\mathrm{LR}$ as the first event was $19 \%$ higher for 1000 units increase in NC and WBCC and $15 \%$ higher for any unitary increase in NLR. Castrated males showed a higher risk of experiencing LR than intact males $(\mathrm{HR}=3.457 ; \mathrm{p}=0.037)$. Acral tumours had a higher probability of LR than PWT located elsewhere $(\mathrm{p}=0.017)$. Histological grade $(\mathrm{p}<0.001)$, necrosis $(\mathrm{p}=0.0028)$ and mitotic count $(\mathrm{p}<0.001)$ were associated with LR: grade II and III $(\mathrm{p}=0.0432 ; \mathrm{p}<0.001)$, necrosis $>50 \%(\mathrm{p}=0.003)$ and each ynitary increase in mitotic index $(\mathrm{p}<0.001)$ were associated with a higher hazard of LR.

Histologically infiltrated margins were prognostic for LR both when including close margins in the infiltrated category $(\mathrm{p}=0.009)$ or in the tumour-free category $(\mathrm{p}=0.002)$. Adjuvant therapies were also associated with LR $(\mathrm{p}<0.001)$, with treated dogs being five times more likely to relapse.

The prognostic effect of each haematological variable (WBCC, NC, LC and NLR) was adjusted for clinical and histopathological variables in multivariate analysis. Models included a maximum of 4 variables each: 1) margins, grade and tumour size; 2) tumour location, size and ulceration; 3) mitotic count and necrosis (Table S3). LC did not have a significant role in any model. WBCC and NC had a significant prognostic role on LR when adjusted for margins, grade, tumour size, location and ulceration (model 1 and 2), but lost their significance when adjusted for mitotic index and necrosis (model 3). NLR remained prognostic for LR only in model 1 when close margins were 
considered as infiltrated. Grade III PWTs had a significantly higher risk of LR than grade I-II tumours when adjusted for margins, size and haematological variables. Incompletely excised PWTs had a significantly higher hazard of LR than tumours excised with free or close margins, independently from the haematological variables, tumour grade and size. PWTs located on the extremities had a significantly higher hazard of LR than PWTs located elsewhere, independently from size, ulceration and haematological variables. When necrosis and mitotic count were considered jointly, PWTs with $>50 \%$ necrosis had a higher risk of LR than PWTs with $<50 \%$ necrosis, and the hazard of LR increased with increasing mitotic index, even when adjusted for haematological variables. Ulceration was a significant risk factor when considered jointly with location, size and haematological variables. Tumour size had no impact on LR.

Finally, in the conjunct analysis of the haematological variables, when considering WBCC, NC and NLR together no association with LR was found (Table S3); WBCC and NC were prognostic for LR while NLR lost its significance in subset models.

\section{Prognostic impact of variables on OST}

In univariate analysis, Boxers had a worse prognosis than mixed-breeds ( $\mathrm{p}=0.019)$; ulceration $(\mathrm{p}=0.016)$, grade III $(\mathrm{p}<0.001)$, necrosis $>50 \%(\mathrm{p}=0.018)$, older age $(\mathrm{p}=0.007)$, increasing mitotic count $(p=0.003)$ were significantly associated with a higher risk of death. None of the leukocyte counts, and NLR were prognostic for survival (Table 2).

In multivariate analysis, Grade III, $>50 \%$ necrosis, higher mitotic count and PWT ulceration were independently significant for OST in all statistical models, while none of the leukocyte counts or NLR had an impact on survival (Table S4). When considering the joint impact on OST of the significant variables of the models, only $>50 \%$ necrosis remained significant (Table S4).

\section{Discussion}


This study investigates pre-treatment leukocyte counts and NLR as prognostic variables for the first time in histologically confirmed canine PWTs undergoing curative-intent surgery. To our knowledge, this is the largest study analysing the prognostic impact of clinical and pathological variables for canine PWTs.

Twenty-seven per cent of dogs experienced LR within two years, and $13 \%$ of dogs died of causes related to tumour progression including distant metastatic disease. The results of this work confirmed previous data on smaller populations ${ }^{2,3}$ and agree with a recent study. ${ }^{23}$ However, in the latter study, the histological and immunohistochemical features of included tumours were not specified, and it is thereby challenging to make the comparisons.

In the current study, the hazard of LR increased with increasing values of pre-treatment WBCC, NC and NLR, while LC did not influence TLR. However, in multivariate analysis WBCC and NC lost their significance when adjusted for mitotic count and tumour necrosis, suggesting a more relevant prognostic role of these histological variables, and NLR remained significant only when adjusted for margin status. Conversely, none of the examined haematological variables was prognostic for OST, while histological parameters seemed to have a substantial prognostic impact on survival. However, these results should be cautiously interpreted since LR represented the main event while tumour-related death was infrequent.

CBC parameters have been widely investigated as potential prognostic markers for several malignancies in humans, and the role of NLR in predicting survival for STS patients is well established. $^{7,24-28}$ MacFarlane and colleagues found that NLR was significantly higher in dogs with STS compared with dogs with benign soft tissue tumours, although neither NLR nor leukocyte counts correlated with tumour grade in the STS group. ${ }^{11}$ Likewise, NLR, WBCC and NC were prognostic for both LR and OST of surgically resected FISS in a recent study, whereas LC did not correlate with any of the endpoints. ${ }^{13}$ Lymphocytes comprise distinctive subpopulations in dogs, and it is reasonable to assume that the lack of prognostic impact of LC on LR is due to a possible influence of specific lymphocyte subsets rather than the absolute lymphocyte count, as previously 
reported in dogs with mammary cancer. ${ }^{29,30}$ On the other hand, the efficacy of the antitumoral immune response may be less influenced by absolute LC in mesenchymal tumour than in other tumour types. Indeed, while most published veterinary literature on the prognostic impact of inflammatory markers focuses on round cell tumours, ${ }^{8,10,12}$ only this and a previous study on FISS ${ }^{13}$ have been conducted on mesenchymal tumours, both reporting a lack of prognostic value of LC. However, future studies on the impact of leukocyte populations on different tumour types are warranted to confirm this result.

When evaluating the concurrent impact of NLR, NC and WBCC on LR, NLR lost its significance, while WBCC and NC remained independently prognostic parameters. This result was unexpected considering that leukocyte ratios are less affected by pathophysiological fluctuations of single leukocyte populations, and NLR has been reported to be a better predictor of outcome than absolute leukocyte counts both in human and canine patients. ${ }^{7,12}$ It is reasonable to assume that incorporation of a non-prognostic LC could have impaired the prognostic value of NLR, similarly to FISS. ${ }^{13}$

Analysis of association measured the correlation between the examined haematological parameters and variables that may act as confounding factors. NLR did not correlate with any of the examined variables; WBCC, NC and LC were all influenced by sex, and WBCC and NC were also associated with the breed, with Boxers having lower counts than the others. Although one paper reported variations in lymphocyte subsets of healthy dogs related to age, gender, and breed, ${ }^{31}$ the effects of patients' variables on the distribution of leukocyte subpopulations have not been unravelled yet and warrant further investigations. Interestingly, none of the haematological variables was affected by concomitant diseases recorded in the study population, suggesting that inclusion of dogs with such comorbidities did not bias a reliable evaluation of the impact of pre-treatment leukocyte counts and NRL (Table 1).

NC was significantly higher in PWTs of grade III, suggesting that NC might be useful to predict a more aggressive clinical behaviour. Indeed, histological grade is an established prognostic factor for $\mathrm{STS}^{32,33}$ and the same conclusion was drawn in the current study. In the future it may be interesting 
to explore whether NC may predict grade in PWTs, thereby identifying dogs that might benefit from aggressive treatments.

Grade, mitotic count and percentage of necrosis were independently prognostic for TLR and OST, with an increased hazard of LR and death for grade III PWTs, $>50 \%$ necrosis and high mitotic index. This is in contrast with previous reports on PWTs, where tumour grade and its components were not statistically associated with prognosis, ${ }^{2,3,23}$ although it is comparable with data on other STSs. ${ }^{32-37}$ This discrepancy may be due to the low number of relapses and fewer grade III PWTs included in the previous studies, which lowered the statistical power and precluded a multivariate analysis. $^{2,3}$

In this case series, infiltrated margins had a higher hazard of LR when compared with tumour-free and close margins both in univariate and multivariate analyses. Interestingly, when close margins were considered together with infiltrated margins, this variable lost its significance in multivariate models, suggesting that close margins may not be at higher risk of LR. ${ }^{38}$ Obtaining tumour-free margins is one of the mainstays of surgical oncology, and completeness of excision is a recognised prognostic factor for several canine malignancies, including STS. ${ }^{34-37}$ However, the role of bistological margins is still controversial for PWTs, with two studies reporting no correlation between margins and LR, although recurrence was observed only in close and infiltrated margins; ${ }^{2-3}$ furthermore, margin status had a prognostic relevance in the analysis of pathological profiles. ${ }^{3}$ This discrepancy is likely due to the smaller sample size of previous studies that reduced the statistical power, since specimen processing and margin evaluation was consistent among studies. Results of this study should draw attention to the importance of margin status for the identification of PWTs at higher risk of recurrence and highlight the importance of adequate surgical planning for PWTs excision aimed at obtaining tumour-free margins.

Among the examined clinical variables, sex and tumour sites were statistically associated with LR in univariate analysis, although only tumour location remained independently prognostic in multivariate models. More specifically, PWTs located on the extremities had a higher hazard of LR 
than PWTs located elsewhere. This agrees with a previous study, where PWTs located at the extremities and infiltrating the muscular layer had the highest hazard of recurrence. ${ }^{3}$ A possible explanation is that a wide surgical excision with tumour-free margins is more challenging to achieve on distal extremities; hence, adjuvant treatment should be probably suggested in these patients.

Tumour ulceration had an independent prognostic impact on both LR and OST. Ulceration has been reported as an adverse prognostic factor for canine MCT, and recently for FISS, although this variable has not been previously evaluated for canine STS. ${ }^{13,40}$ Neoplastic ulceration occurs due to the rapid tumour growth which impair tissues vascularisation or to cutaneous infiltration from slow growing tumours, and it is thus reasonable to assume that this feature correlates with a more aggressive biological behaviour.

Age and gender correlated with OST and LR, respectively, in univariate analysis. However, they lost the significance in multivariate models. The impact of age on survival is easily understood, as older dogs are more likely to experience a fatal outcome during the follow-up period (70\% of included dogs died of tumour-unrelated causes). On the other hand, further studies should clarify the relationship between gender and the hazard of LR, considering the absence of data in the literature reporting a possible hormonal influence in canine PWT and STS.

Surprisingly, histological growth pattern and tumour size were not prognostic for LR nor OST. Again, this result differs from previous studies on PWT and STS., ${ }^{2,33}$ According to the existing literature, increasing tumour size was significantly associated with an increased hazard of LR, and PWTs invading the muscular layer recurred more frequently., ${ }^{2,3}$ Our result may be due to the effect of more robust prognostic variables in multivariate analysis, such as grade, necrosis, mitotic index and surgical margins.

Last, the impact of adjuvant treatments was assessed in univariate analysis, although the low number of events precluded the inclusion of this variable in multivariate models. Dogs receiving adjuvant chemotherapy or radiation therapy were five times more likely to experience LR during 
the follow-up period. It may be conceivable that dogs with one or more negative prognostic factors were more likely to receive adjuvant treatments. To date, there are no reports available on the efficacy of adjuvant treatments on canine PWTs. Adjuvant radiation therapy seems to improve local control rates of surgically excised canine STS, although evidence is still limited to a few retrospective studies without a control group. ${ }^{41-43}$ Conversely, dogs with STS treated with adjuvant intravenous chemotherapy did not show a significant improvement in outcome, ${ }^{44}$ although promising results have been reported in one study with the use of metronomic chemotherapy. ${ }^{45} \mathrm{~A}$ pathological study reported the expression of VEGF-, PDGF- and hEGE-mediated pathways in canine PWTs indicating their receptors as possible therapeutic targets, although clinical studies are lacking. ${ }^{46}$

This study has some limitations, mainly related to its retrospective nature and to the low number of events (LR and tumour-related death) that precluded a multivariate analysis, thus only the conjunct impact of the more clinically relevant variables was evaluated. Furthermore, the paucity of recurrences recorded in this population hampered the identification of optimal cut-off values for the haematological variables. However, this study has the merit of reporting the largest cohort of dogs vvith histologically confirmed PWTs: the inclusion of a homogeneous group indeed limited the study bias and allowed for precise assessment of the prognostic impact of haematological, clinical and pathological features.

In conclusion, pathological variables such as grade, percentage of necrosis, mitotic count and status of surgical margins had an impact on LR in this large cohort of canine PWTs and need to be considered to predict the prognosis more accurately. Moreover, it is interesting to mirror that pretreatment leukocyte counts and NLR may represent useful parameters assisting the identification of those PWTs at higher risk of LR. If confirmed in future prospective studies, inclusion of such variables in the pre-operative evaluation of canine PWTs may allow to plan a more extensive surgery when feasible and/or adjuvant treatments in selected dogs. Conversely, the lack of impact 
of growth pattern and tumour size underlines the need to better assess the conjunct impact of pathological variables on the outcome of canine PWTs on a broader sample.

\section{References}

1. Avallone G, Helmbold P, Caniatti M, et al. The spectrum of canine cutaneous perivascular wall tumors: morphologic, phenotyping and clinical characterization. Vet Pathol 2007;44:607-20.

2. Stefanello D, Avallone G, Ferrari R, et al. Canine cutaneous perivascular wall tumors at first presentation: clinical behaviour and prognostic factors in 55 dogs. $J$ Vet Intern Med 2011;25:1398-405.

3. Avallone G, Boracchi P, Stefanello D, et al. Canine perivascular wall tumors: high prognostic impact of site, depth and completeness of margins. Vet Pathol 2014;51:713-21.

4. Avallone G, Stefanello D, Ferrari R, Roccabianca P. The controversial histologic classification of canine subcutaneous whorling tumors: the path to perivascular wall tumors. Vet Comp Oncol 2020;18:3-8.

5. Borgatti A, Dickerson EB, Lawrence J. Emerging therapeutic approaches for canine sarcomas: pushing the boundaries beyond the conventional. Vet Comp Oncol 2020;18:9-24.

6. Coussens LM, Webr Z. Inflammation and cancer. Nature 2002;420:860-7.

7. Templeton AJ, McNamara MG, Šeruga B,et al. Prognostic role of neutrophil-to-lymphocyte ratio in solid tumors: a systematic review and meta-analysis. $J$ Natl Cancer Inst 2014;106(6):1-11. 
8. Marconato L, Martini V, Stefanello D, et al. Peripheral blood lymphocyte/monocyte ratio as a useful prognostic factor in dogs with diffuse large B-cell lymphoma receiving chemoimmunotherapy. Vet J 2015 ;206(2):226-230.

9. Sottnik JL, Rao S, Lafferty MH, et al. Association of blood monocyte and lymphocyte count and disease-free interval in dogs with osteosarcoma. J Vet Intern Med 2010;24(6):14391444.

10. Macfarlane MJ, Macfarlane LL, Scase T, Parkin T, Morris JS. Use of neutrophil to lymphocyte ratio for predicting histopathological grade of canine mast cell tumours. Vet Rec 2016 12;179(19):491-503.

11. Macfarlane L, Morris J, Pratschke K, et al. Diagnostic value of neutrophil-lymphocyte and albumin-globulin ratios in canine soft tissue sarcoma. J Small Anim Pract 2016;57(3):135141.

12. Skor O, Fuchs-Baumgartinger A, Tichy A, Kleiter M, Schwendenwein I. Pretreatment leukocyte ratios and concentrations as predictors of outcome in dogs with cutaneous mast cell tumours. Vet Comp Oncol 2017;15(4):1333-1345.

13. Chiti LE, Martano M, Ferrari R, et al. Evaluation of leukocyte counts and neutrophil-tolymphocyte ratio as predictors of local recurrence of feline injection site sarcoma after curative intent surgery. Vet Comp Oncol 2020;18:105-116.

14. Stefanello D, Morello E, Roccabianca P, et al. Marginal excision of low-grade spindle cell sarcoma of canine extremities: 35 dogs (1996-2006). Vet Surg 2008;37:461-5.

15. Bray JP. Soft tissue sarcoma in the dog - part 2: surgical margins, controversies and a comparative review. J Small Anim Pract 2017;58:63-72.

16. Kuntz CA, Dernell WS, Powers BE, et al. Prognostic factors for surgical treatment of softtissue sarcomas in dogs: 75 cases (1986-1996). J Am Vet Med Assoc 1997;211:1147-51. 
17. Stromberg PC, Meuten DJ. Trimming Tumors for Diagnosis and Prognosis. In: Donald J. Meuten ed., Tumors in Domestic Animals. 5th ed., Ames, Iowa: Wiley Blackwell; 2017: 3551.

18. Beyerlein A. Quantile regression—opportunities and challenges from a user's perspective. American journal of epidemiology 2014;180:330-331.

19. Schemper M, Smith TL. A note on quantifying follow-up in studies of failure time. Controlled clinical trials 1996;17:343-346.

20. Kim HT. Cumulative incidence in competing risks data and competing risks regression analysis. Clinical Cancer Research 2007;13:559-565.

21. Peduzzi, P, Concato, J, Feinstein AR, Holford TR. Importance of events per independent variable in proportional hazards regression analysis II. Accuracy and precision of regression estimates. Journal of clinical epidemiology, 1995;48:1503-151

22. Vittinghoff, E., \& McCulloch, C. E. (2007). Relaxing the rule of ten events per variable in logistic and Cox regression. American journal of epidemiology, 165(6), 710-718

23. Kravitz A, Davis G, Bastian RP, Fittipaldi K. Outcome and prognostic indicators for hemangiopericytomas in dogs: 167 cases (2009-2016). J Am Anim Hosp Assoc 2019;55:194200.

24. Idowu OK, Ding Q, Taktak AFG, Chandrasekar CR, Yin Q. Clinical implication of pretreatment neutrophil to lymphocyte ratio in soft tissue sarcomas. Biomarkers 2012;17:539-544.

25. Szkandera J, Gerger A, Liegl-Atzwanger B, et al. The derived neutrophil/lymphocyte ratio predicts poor clinical outcome in soft tissue sarcoma patients. Am J Surg 2015;210(1):111116.

26. Kobayashi H, Okuma T, Oka H, et al. Neutrophil-to-lymphocyte ratio after pazopanib treatment predicts response in patients with advanced soft-tissue sarcoma. Int J Clin Oncol 2018;23:368-374. 
27. Liu G, Ke LC, Sun SR. Prognostic value of pretreatment neutrophil-to-lymphocyte ratio in patients with soft tissue sarcoma: a meta-analysis. Medicine (Baltimore) 2018;97:e12176.

28. Mirili c, Paydas S, Guney IB, et al. Assessment of potential predictive value of peripheral blood inflammatory indexes in 26 cases with soft tissue sarcoma treated with pazopanib: a retrospective study. Cancer Manag Res 2019;11:3445-3453.

29. Estrela-Lima A, Araújo MS, da Costa-Neto JM, et al. Understanding of the immunological heterogeneity of canine mammary carcinomas to provide immunophenotypic features of circulating leukocytes as clinically relevant prognostic biomarkers. Breast Cancer Res Treat 2012;131(3):751-763.

30. Karayannopoulou M, Anagnostou T, Margariti A, et al. Evaluation of blood T-lymphocyte subpopulations involved in host cellular immunity in dogs with mammary cancer. Vet Immunol Immunopathol 2017;186:45-50.

31. Faldyna M, Levà L, Knotigovà $\mathrm{P}$, Toman M. Lymphocyte subsets in peripheral blood of dogs—a flow cytometric study. Vet Immunol Immunopathol 2001;82:23-37.

32. McSporran KD. Histologic grade predicts recurrence for marginally excised canine subucutaneous soft tissue sarcomas. Vet Pathol 2009;46:928-33.

33. Bray JP, Polton GA, McSporran KD, et al. Canine soft tissue sarcoma managed in first opinion practice: outcome in 350 cases. Vet Surg 2014;43:774-82.

34. Ehrhart N. Soft-tissue sarcomas in dogs: a review. J Am Anim Hosp Assoc 2005;41:241-6.

35. Dennis MM, McSporran KD, Bacon NJ, et al. Prognostic factors for cutaneous and subcutaneous soft tissue sarcomas in dogs. Vet Pathol 2011;48:73-84.

36. Hohenhaus AE, Kelsey JL, Haddad J, et al. Canine cutaneous and subcutaneous soft tissue sarcoma: an evidence-based review of case management. J Anim Hosp Assoc 2016;52:7789. 
37. Millovancev M, Townsend KL, Tuohy JL, et al. Long-term outcomes of dogs undergoing surgical resection of mast cell tumors and soft tissue sarcomas: a prospective 2-year-long study. Vet Surg 2020;49:96-105.

38. Liptak JM. Histologic margins and the residual tumour classification scheme. Vet Comp Oncol 2020; doi:10.1111/vco.12620.

39. Milovancev M, Tuohy JL, Townsend KL, Irvin V. Influence of surgical margin completeness on risk of local tumour recurrence in canine cutaneous and subcutaneous soft tissue sarcoma: a systematic review and meta-analysis. Vet Comp Oncol 2019;17:354-364.

40. Stefanello D, Buracco P, Sabattini S, et al. Comparison of 2- and 3- category histologic grading systems for predicting the presence of metastasis at the time of initial evaluation in dogs with cutaneous mast cell tumours: 368 cases (2009-2014). J Am Vet Med Assoc 2015;246:765-9.

41. Forrest LJ, Chun R, Adams WM. Postoperative radiotherapy for canine soft tissue sarcoma. $J$ Vet Intern Med 2000;14:578-82.

42. McKnight JA, Mauldin GN, McEntee MC, Meleo KA, Patnaik AK. Radiation treatment for incompletely excised resected soft-tissue sarcomas in dogs. J Am Vet Med Assoc 2000;217:205-10.

43. Demetriou JL, Brearley MJ, Constantino-Casas F, Addington C, Dobson J. Intentional marginal excision of canine limb soft tissue sarcomas followed by radiotherapy. J Small Anim Pract 2012;53:174-81.

44. Bray JP. Soft tissue sarcoma in the dog - Part 1: a current review. J Small Anim Pract 2016;57:510-519.

45. Elmslie RE, Glawe P, Dow SW. Metronomic therapy with cyclophosphamide and piroxicam effectively delays tumor recurrence in dogs with incompletely resected soft tissue sarcomas. $J$ Vet Intern Med 2008;22:1373-9. 
46. Avallone G, Stefanello D, Boracchi P, et al. Growth factors and COX2 expression in canine perivascular wall tumors. Vet Pathol 2015;52:1034-40.

\section{Supporting information}

Tables S1-S4 may be found online in the supporting information section at the end of the article. 


\section{Figure Legends}

\section{Figure 1}

Cumulative incidence of local relapse estimated by the method for competing risks (i.e. the probability of observing local recurrence as the first event). The occurrence of death without a local recurrence occurred before death is the competing risk. Continuous line represents the cumulative incidence of local recurrence and dotted lines the $95 \%$ confidence intervals.

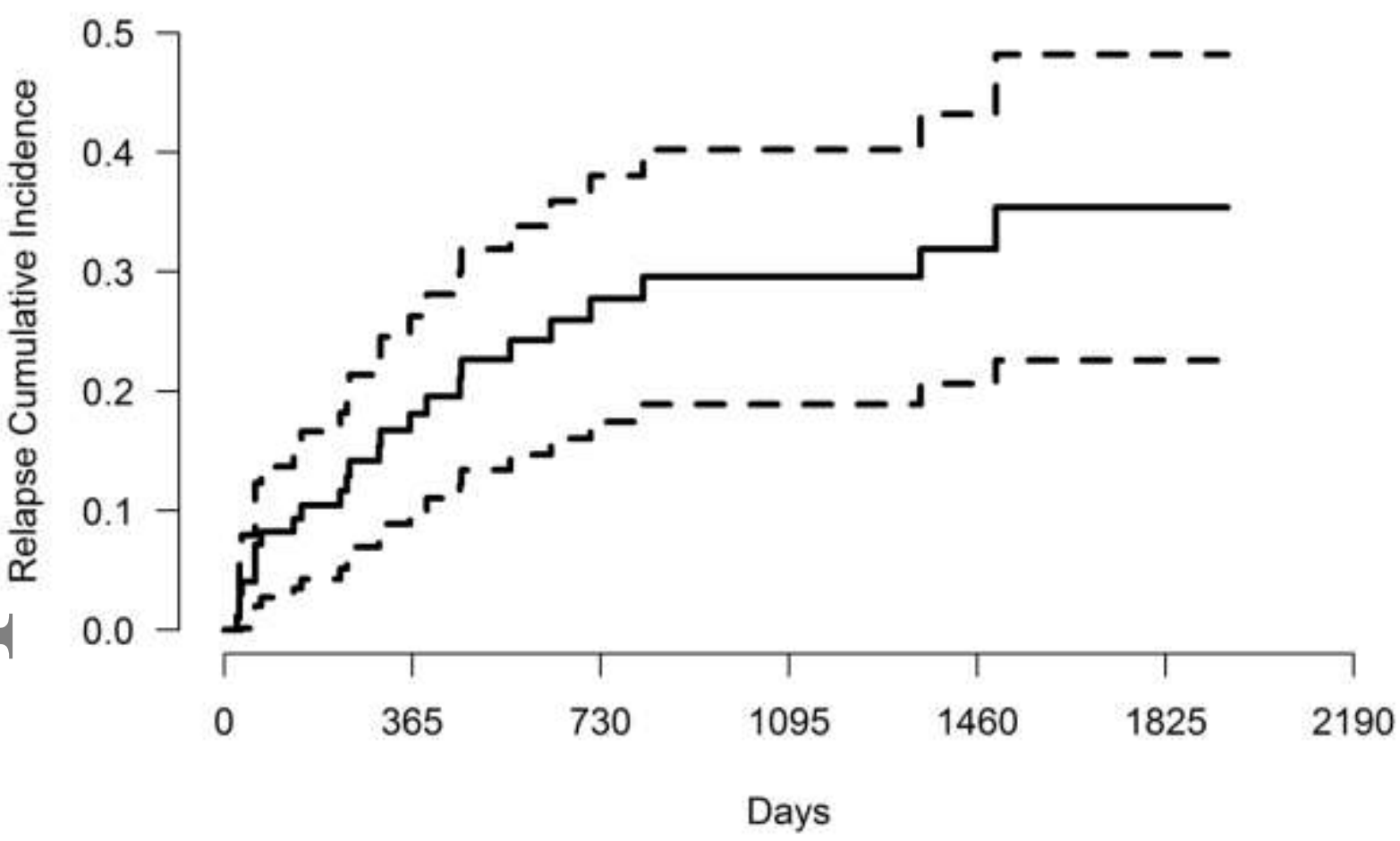

Figure 2

Kaplan-Meier estimated survival probability (continuous line) and 95\% confidence intervals (dotted lines). Vertical lines correspond to censored cases. 
${ }^{\dagger}$ Wald test. For variables with more than two categories Wald test for overall contribution is also reported ( $\mathrm{p}$-value in gray shaded lines)

${ }^{\mathbb{T}}$ Gray test was-applied for overall variable effect because no local recurrence was reported in boxers. *Statistically significant at $5 \%$ level.

WBCC=white blood cell count; NC=neutrophil count; LC=lymphocyte count; NLR=neutrophil-to-lymphocyte ratio

Table 2. Univariate analysis of clinical and pathological and haematological variables on overall

survival: results of Cox regression model. For categorical variables results are reported as the ratio of the hazard of death for each category to the hazard of death in the reference category. For continuous variables results are reported as the hazard ratio for the unit increase.

\begin{tabular}{|l|l|l|l|}
\hline Variable & Hazard ratio & $\mathbf{9 5 \%}$ C.I. & $\boldsymbol{P}^{\dagger}$ \\
\hline BREED & & & 0.1706 \\
\hline Boxer $v s$ mixed-breed & 3.372 & $1.220-9.319$ & $0.019^{*}$ \\
\hline Labrador $v$ mixed-breed & 1.446 & $0.529-3.950$ & 0.472 \\
\hline Other $v s$ mixed-breed & 0.982 & $0.482-2.002$ & 0.960 \\
\hline SEX & & & 0.1228 \\
\hline Castrated male $v$ Intact male & 1.834 & $0.738-4.559$ & 0.191 \\
\hline Intact female $v$ Intact male & 0.443 & $0.143-1.369$ & 0.157 \\
\hline Spayed female $v$ Intact male & 1.106 & $0.512-2.387$ & 0.798 \\
\hline TUMOR LOCATION & & & 0.3229 \\
\hline Extremities $v s$ Others & 1.026 & $0.53-1.983$ & 0.94 \\
\hline ULCERATION & & & \\
\hline Present $v s$ Absent & 3.976 & $1.527-10.353$ & $0.005^{*}$ \\
\hline OONC. DISEASE & & & \\
\hline Present $v s$ Absent & 1.43 & $0.767-2.666$ & 0.26 \\
\hline GRADING & & & $<0.0001^{*}$ \\
\hline G II $v$ G I & 1.191 & $0.581-2.439$ & 0.633 \\
\hline G III $v s$ GI & 20.976 & $7.056-62.363$ & $<0.0001^{*}$ \\
\hline NECROSIS & & & $0.01841^{*}$ \\
\hline$<50 \% v s$ Absent & 1.535 & $0.769-3.063$ & 0.224 \\
\hline$>50 \%$ Absent & 6.814 & $2.164-21.459$ & $0.001^{*}$ \\
\hline PATTERN OF GROWTH & & & \\
\hline Infiltrative $v s$ Espansile & 1.21 & $0.615-2.38$ & 0.581 \\
\hline SURGICAL MARGINS & & & 0.5014 \\
\hline Infiltrated+Close $v s$ Tumor-free & 1.247 & $0.651-2.386$ & 0.506 \\
\hline Infiltrated $v s$ Tumore-free+Close & 1.184 & $0.637-2.2$ & 0.593 \\
\hline ADJUVANT TRETMENT & & & \\
\hline Yes $v s$ No & 1.166 & $0.556-2.447$ & 0.685 \\
\hline AGE & & & \\
\hline for 1 year increase & 1.251 & $1.060-1.477$ & $0.008^{*}$ \\
\hline WEIGHT & & & 0.518 \\
\hline for 1 kg increase & 1.009 & $0.981-1.038$ & \\
\hline TUMOR SIZE & 1.078 & $0.998-1.164$ & 0.057 \\
\hline for 1 cm increase & & & 0.369 \\
\hline WBCC & 1.046 & $0.948-1.154$ & \\
\hline for 1000 cells increase & & & \\
\hline NC & & & \\
\hline
\end{tabular}




\begin{tabular}{|l|l|l|l|}
\hline for 1000 cells increase & 1.057 & $0.953-1.173$ & 0.297 \\
\hline LC & & & \\
\hline for 1000 cells increase & 1.067 & $0.677-1.682$ & 0.781 \\
\hline NLR & & & \\
\hline for 1 unit increase & 0.996 & $0.874-1.135$ & 0.95 \\
\hline MITOTIC COUNT & & & \\
\hline for 1 unit increase & 1.055 & $1.018-1.093$ & $0.003^{*}$ \\
\hline
\end{tabular}

${ }^{1}$ Wald test. For variables with more than two categories overall test is also reported (p-value in gray shaded line).*Statistically significant at $5 \%$ level.

WBCC=white blood cell count; $\mathrm{NC}=$ neutrophil count; $\mathrm{LC}=$ lymphocyte count; NLR=neutrophil-tolymphocyte ratio

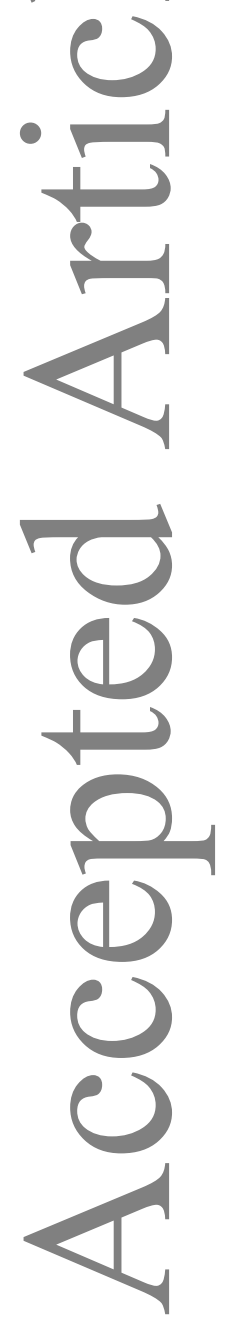

